\title{
Anabases
}

ANABASES Traditions et réceptions de l'Antiquité

$14 \mid 2011$

Varia

\section{Introduction. Brumoy, pédagogue et passeur du théâtre grec}

\section{Malika Bastin-Hammou}

\section{(2) OpenEdition}

1 Journals

Édition électronique

URL : http://journals.openedition.org/anabases/2137

DOI : 10.4000/anabases. 2137

ISSN : 2256-9421

Éditeur

E.R.A.S.M.E.

\section{Édition imprimée}

Date de publication : 1 octobre 2011

Pagination : 27-41

ISSN : 1774-4296

\section{Référence électronique}

Malika Bastin-Hammou, «Introduction. Brumoy, pédagogue et passeur du théâtre grec », Anabases [En ligne], 14 | 2011, mis en ligne le 01 octobre 2014, consulté le 20 octobre 2019. URL : http:// journals.openedition.org/anabases/2137; DOI : 10.4000/anabases.2137 
Anabases 14 (2011), p. 27-41.

\section{Introduction. \\ Brumoy, pédagogue \\ et passeur du théâtre grec}

Malika Bastin-Hammou

EN 1730 paraît à Paris le Théatre des Grecs du jésuite Pierre Brumoy, maître de rhétorique au collège Louis-le-Grand ${ }^{1}$. L'ouvrage - mille cinq cents pages en trois volumes in quarto - propose des traductions en prose, intégrales ou anthologiques, des pièces d'Eschyle, Sophocle, Euripide et Aristophane, c'est-à-dire de l'intégralité du théâtre grec connu à cette date. Les traductions sont précédées de trois discours Discours sur le théâtre des Grecs, sur l'Origine de la Tragédie, sur le Parallèle du Théâtre ancien et moderne. Le premier expose le projet, le second contextualise le théâtre grec et le troisième souligne les principales différences entre le théâtre antique et le théâtre moderne. Il s'agit en effet pour Brumoy d'amener ses lecteurs à découvrir le théâtre grec munis des connaissances historiques nécessaires à sa compréhension et de les mettre en garde contre une attitude qui consisterait à l'apprécier à l'aune du théâtre moderne. Les traductions qui suivent sont chacune précédées d'une introduction, accompagnées de notes et suivies d'une part de commentaires visant à renseigner le lecteur sur leur contexte et d'autre part de pièces latines, françaises et étrangères proposées à titre de comparaison. L'Edipe de Sophocle, qui ouvre le premier tome, est ainsi suivi de ceux de Sénèque, Corneille et Giustiniano.

L'objectif premier de ces traductions est de donner accès au public lettré, mais ne lisant pas le grec, au répertoire tragique et comique des Grecs. Mais il s'agit aussi, dans un contexte toujours marqué par la Querelle, de faire connaitre ce théâtre injustement

1 Le Théâtre des Grecs, par le R. P. Brumoy, de la Compagnie de Jésus, à Paris, chez Rollin Père, Jean-Baptiste Coignard et Rollin fils, 1730. 
décrié, voire de revivifier la production théâtrale française alors en crise en amenant les dramaturges en mal d'inspiration "à la source".

Dans la pratique, Brumoy est allé moins loin qu'il ne le dit : il ne traduit intégralement que sept tragédies, et aucune d'Eschyle. L'écho de son ouvrage n'en est pas moins considérable. La parution du Théatre des Grecs marque en effet durablement l'histoire de la réception du théâtre antique. Traduit en anglais ${ }^{2}$, il est diffusé dans toute l'Europe - Schlegel en recommande la lecture pour la formation des comédiens ${ }^{3}$. Mais c'est surtout en France qu'il devient, et pour longtemps, l'ouvrage de référence sur le théâtre grec. Comme le souhaitait Brumoy, son travail donne une véritable impulsion aux dramaturges de son temps qui, à sa suite, s'intéressent à des pièces délaissées - comme l'Iphigénie en Tauride d'Euripide.

Si la critique s'est ponctuellement intéressée à la figure du P. Brumoy, en analysant par exemple sa conception de Sénèque ou de Racine, ou encore son influence sur les dramaturges allemands, il n'y a à ce jour que peu de travaux portant sur l'ensemble du Théâtre des Grecs. Voilà pourquoi nous avons voulu consacrer une journée d'étude à la figure intellectuelle de Brumoy et à son ouvrage majeur, le Théatre des Grecs, en privilégiant la perspective de l'histoire de la réception du thêâtre antique, qu'elle a durablement marquée. Quel est l'objet de cet ouvrage singulier ? Quelles pièces Brumoy choisit-il de traduire, et pourquoi ? Quelle est sa conception, quelles sont ses pratiques de traduction ? Quelle est la place de la scène dans son appréhension de ce théâtre ? Quelle est l'influence des débats de son temps ? Quelles furent, enfin, la diffusion et la postérité de son ouvrage ? Telles sont les questions auxquelles tentent de répondre les travaux ici réunis.

\section{Le Père Brumoy (1688-1742) et le théâtre grec en 1730}

Le P. Brumoy est un jésuite, né à Rouen le 26 août 1688. Historien, poète, traducteur, il fut peut-être avant tout un pédagogue ${ }^{4}$.

D'abord professeur d'humanités à Caen, puis de rhétorique à Bourges, ensuite précepteur du prince de Talmont, il sera régent de mathématiques à Paris, au collège Louis-le-Grand et ne cessera d'enseigner qu'en 1732, pour se consacrer à l'histoire. Mais cette activité d'enseignement ne doit pas être considérée comme secondaire : le

2 The Greek Theatre offather Brumoy, translated by Mrs Charlotte Lennox, London, printed for Milar, 1759 et Gray, 1985.

3 J.-M. VAlEnTin, “ "Un système [...] faux [...], mais d'où résultent de si grandes beautés”. Tragédie, tragique et destin dans le drame de tradition catholique : le Théâtre des Grecs de Pierre Brumoy s.j. ", dans L'École, la ville, la cour. Pratiques sociales, enjeux poétologiques et répertoires du théâtre dans l'Empire au XVII siècle, Paris, Klincksieck, 2004, p. 173-191.

4 J.-C. Polet (sous la direction de), Patrimoine littéraire européen : anthologie en langue française, de Boeck Université, Bruxelles, 2000, vol. 13. 
pédagogue, maître de rhétorique autant que régent de mathématique, est bien présent dans l'entreprise de vulgarisation systématique que représente le Théâtre des Grecs.

Historien, Brumoy l'est par sa participation aux travaux de plusieurs de ses confrères. Il devient à partir de 1734 un des rédacteurs ordinaires des Mémoires de Trévoux; il collabore aux Révolutions d'Espagne du père d'Orléans (Paris, 1734) et à l'Histoire de l'église gallicane commencée par Jacques Longueval et Pierre-Claude Fontenay (Paris, 1744), en rédigeant les tomes XI et XII. Il est aussi poète : dès 1722 il se fait connaître par des Pensées sur la décadence de la poésie latine et on lui doit un Recueil de diverses pièces en prose et en vers contenant des discours, trois tragédies, deux poèmes latins et deux comédies en vers jouées dans les collèges ${ }^{5}$. Enfin Brumoy, en bon pédagogue, est aussi traducteur, notamment d'ouvrages rédigés en latin par ses confrères. Il traduit ainsi plusieurs discours du Père Porée, dont le De Theatro, La Vie de l'impératrice Éléonor, mère de Charles VI, du Père Wagner (1723) ou encore l'Histoire de Rienzi du père Du Cerceau (1733).

Mais on lui doit surtout le Théâtre des Grecs, où il donne la mesure de ses qualités conjuguées de pédagogue, d'historien, de traducteur et de poète. Avec cette somme, Brumoy a pour ambition de présenter au public, pour la première fois dans son intégralité, ce théâtre selon lui méconnu et mal jugé : "On a peu connu, et [...] l'on ne connaît presque plus le Théâtre des Grecs ", écrit-il dans les premières lignes de son Discours sur le Théâtre des Grecs ${ }^{6}$. Le projet part en effet d'un constat : les Tragiques grecs souffrent d'une méconnaissance injustifiée et qui leur est spécifique : "Il n'en a pas été ainsi de la Morale, de l'Éloquence, de l'Histoire \& de la Poësie. Les Anciens qui nous en ont laissé des modèles, ont picqué beaucoup plus la curiosité des François... Ces fondateurs du théâtre ont le plus souffert de la guerre qui dure encore entre les Anciens \& les Modernes. Le mérite des Historiens, des Orateurs \& des Poëtes s'est fait jour à travers les nuages ; \& celui des Tragiques n’a pû entièrement dissiper les ténèbres qui les enveloppent ${ }^{7}$."

En entreprenant ainsi de rendre justice au théâtre grec, il répond à un appel formulé depuis plusieurs décennies, et auquel certains érudits comme les Dacier avaient commencé à répondre. À la fin du XVII ${ }^{\mathrm{e}}$ siècle, en effet, se fait jour la demande d'une redécouverte des textes anciens tombés dans l'oubli, et notamment du théâtre antique. Certes, plusieurs tragédies avaient été traduites en français dès la Renaissance : dès 1537, Lazare de Baif traduit l'Électre de Sophocle ; en 1544 Bochetel traduit l'Hécube d'Euripide ; Sébillet son Iphigénie à Aulis en 1549 et Jean-Antoine de Baïf l'Antigone de Sophocle en 1573. Mais, entre Jean-Antoine de Baïf et André Dacier, qui en 1692 publie sa traduction d'Edipe-roi et Électre de Sophocle, pendant cent vingt ans donc, aucune tragédie grecque ne paraît plus avoir été traduite en français, ou du moins

5 Recueil de divers ouvrages en prose et en vers, Paris, Rollin fils, 1741.

6 Discours sur le théâtre des Grecs, p. I.

7 Ibid., p. II. 
avoir été publiée. Cette époque ne semble s’intéresser qu’à Sénèque, comme le constate Marie Delcourt ${ }^{8}$.

Les choses sont un peu différentes pour la comédie. L'édition princeps des textes d'Aristophane date de la Renaissance ; on la doit à l'éditeur vénitien Alde Manuce et au savant crétois Marc Musurus (1470-1517) ${ }^{9}$. Suit le temps de l'Aristophane latin, qui débute en fait avant même l'édition princeps, puisqu'en 1440 l'humaniste florentin Leonardo Bruni traduit en latin les deux cent soixante-neuf premiers vers du Ploutos. Mais c'est en 1538 qu'Aristophane devient intégralement accessible au public qui ne sait pas le grec, grâce à la traduction latine de l'humaniste italien Andrea Divo de Capodistria, publiée à Venise, chez Borgofranco ${ }^{10}$. Elle est en partie reprise dans l'Aristophane latin que propose en 1586, à Francfort, Nicodemus Frischlin, puis dans l'édition bilingue d'Edouard Bizet de Charlais et Aemilius Portus, publiée à Genève en 1607, dont les traducteurs sont Divo (Oiseaux, Thesmophories, Assemblée des femmes), Frischlin (Ach. Eq. Nub. Ran. Plut) et Florent Chrestien (La Paix ${ }^{11}$, Les Guêpes, Lysistrata). En 1624 paraît un Aristophane latin sous la direction de Scaliger, dû à plusieurs mains. Enfin en 1670 Tanneguy Le Fèvre fait paraitre à Amsterdam un Aristophane latin où l'on retrouve les traductions de Divo, Frischlin, Chrestien... et de Tanneguy le Fèvre lui-même, puisqu'y figure sa traduction de l'Assemblée des femmes, qu'il avait déjà publiée seule en 1659 (reprint 1665 et 1674).

Les traductions de Frischlin, Chrestien et Tanneguy le Fèvre ont toutes en commun de présenter le latin et le grec sur des pages opposées, ce qui laisse supposer qu'elles avaient pour objet peut-être autant de donner accès à Aristophane que de diffuser la connaissance du grec. Les traductions en français, sans texte grec et donc visant spécifiquement à faire connaître l'œuvre du poète comique, se font donc attendre bien plus longtemps pour la comédie que pour la tragédie. Certes, en 1560, Jean-Antoine de Baiff aurait traduit en français le Ploutos - mais le texte est aujourd'hui perdu. On dispose bien de quelques vers du Ploutos traduits en vers français par Ronsard, mais l'attribution de ce court texte pose problème ${ }^{12}$. Enfin en 1579, Pierre le Loyer, un juris-

8 M. Delcourt, La tradition des comiques anciens en France avant Molière, Paris, Droz, 1934.

9 Pour plus de détails sur la réception d'Aristophane depuis l'Antiquité et notamment des Nuées, voir M. Bastin-Hammou, "La réception française des Nuées d'Aristophane: quelques jalons ", dans C. Cusset et M.-P. NoËL, Contribution à l'étude des Nuées d'Aristophane, Cahiers du Groupe Interdisciplinaire de Théâtre Antique, à paraître.

10 Aristophanis comicorums principis comoediae undecim e graeco in latinum, ad verbum, translatae; Andrea Divo Justinopolitano interprete, Venetiis apud D. Jacob a Burgofrancho Papiensem, Mense Junio M.D.XXXVIII.

11 Sa traduction de la Paix avait déjà été publiée seule en 1588-89. Il s'agit donc ici d'une reprise.

12 L'authenticité de ce fragment a été contestée : il ne figure dans les œuvres de Ronsard que depuis l'édition de 1617. On a discuté également sur le point de savoir si Ronsard avait traduit l'œuvre entière ou seulement ces 264 vers. Surtout, parler ici de traduction pose 
consulte toulousain zélateur de Rabelais compose, à partir des Oiseaux d'Aristophane, une Néphélococugie, mais elle est fort éloignée de l'original ${ }^{13}$. Entre cet Aristophane latin et ces rares essais de passage en français, les comédies d'Aristophane ne sont donc, pendant longtemps, pas accessibles au grand public. Ce n'est en fait qu'en 1684 qu'Anne Le Fèvre, la fille de Tanneguy le Fèvre et future Anne Dacier, traduit le Ploutos et les Nuées, animée d'un souci de réhabilitation ${ }^{14}$. Ce souci, on le retrouve, peut-être plus nuancé, chez le P. Brumoy ${ }^{15}$ : Romain Piana montre en effet dans les pages qui suivent que Brumoy impose une réévaluation de la fonction de la comédie ancienne, " qui subordonne les attaques personnelles à leur fonction sociale et politique, liée aux principes égalitaires de la démocratie athénienne ». Aristophane n’apparaît plus comme un poète satiriste responsable de la mort de Socrate, mais comme un pilier de la démocratie athénienne.

C'est donc dans ce mouvement de redécouverte des Anciens qui se fait jour dans le grand public à la fin du XVII ${ }^{\mathrm{e}}$ siècle et auquel répondent d'abord la collection ad usum Delphini et les travaux des Dacier que s'inscrit l'entreprise de Brumoy ${ }^{16}$. Il s'en distingue cependant à plusieurs égards, par son refus de l'érudition et son ambition à l'exhaustivité.

L'ouvrage se présente en effet comme une somme. Il est composé de trois volumes et se divise en trois parties, sans que les parties ne correspondent aux volumes : la première partie occupe en effet le premier et le début du deuxième volume. Il réunit

problème. S'il y a bien des bribes de traduction, on est loin de la traduction philologique telle qu'elle se pratique à l'époque - en général en latin. Voir M. Delcourt, Étude sur les traductions des tragiques grecs et latins en France depuis la Renaissance, Bruxelles, Lamertin, 1925 et Ronsard, Euvres complètes. I; édition établie, présentée et annotée par J. Céard, D. Ménager et M. Simonin, Paris, Gallimard, Bibliothèque de la Pléiade, 1991, p. 271-272.

13 La Néphélococugie ou La nuée des cocus : première adaptation des "Oiseaux » d'Aristophane en français, par Pierre Le LoYer ; édition critique préparée par M. Doe et K. CAMERON, Genève, Droz, 2004. Le sujet est cependant différent de celui de l'original, puisqu'il repose sur un jeu de mot : la cité aérienne d'Aristophane, conçue pour les oiseaux, l'est désormais pour les cocus - terme qui désigne aussi des oiseaux, les coucous, au XVI siècle. Les deux Athéniens d'Aristophane quittaient leur cité pour des raisons politiques ; ils deviennent chez Le Loyer deux Toulousains cocus en quête d'un monde meilleur, où les femmes seraient fidèles.

14 Voir, sur ces traductions d'Anne Le Fèvre, M. Bastin-Hammou, «Anne Dacier et les premières traductions françaises d'Aristophane : l'invention du métier de femme philologue ", Littératures classiques 72 (2010), p. 85-99.

15 E. Flamarion, "Que faire d'Aristophane quand on est jésuite en 1730 ? Ou le Théâtre des Grecs du P. Brumoy ", Cahiers du Groupe Interdisciplinaire de Théâtre Antique, "La tradition créatrice du théâtre antique. II. De Rome à nos jours ", 12 (1999).

16 L'Edipe et l'Électre de Sophocle, tragedies grecques traduites en François, avec des remarques, par André Dacier, Chez Claude Barbin sur le second Perron de la sainte Chapelle, Paris, 1692. 
dans ses deux premiers volumes les traductions complètes de trois tragédies de Sophocle et quatre d'Euripide, et des analyses des autres tragédies des trois auteurs. Le troisième volume est consacré à Aristophane et au drame satyrique.

Le premier volume s'ouvre sur trois discours préliminaires : le Discours sur le théatre des Grecs (I-XXVIII), le Discours sur l'origine de la tragédie (XXIX-XCVIII) et le Discours sur le parallèle des théatres (XCIX-CLX). Cette " triple préface ", comme la nomme le jésuite, est une véritable propédeutique : elle vise à " préparer les esprits, sans vouloir les surprendre ${ }^{17}$ ". Le premier discours consiste ainsi en une introduction générale, explicitant le projet et la méthode adoptée pour aborder ce théâtre et lui rendre justice. Le deuxième retrace l'histoire de la tragédie grecque depuis les origines. Le troisième s'attache enfin à mettre en garde ses lecteurs contre le préjugé qui risque de leur faire mesurer le thêâtre des Grecs à l'aune du théâtre moderne : "Comme le préjugé légitime en faveur de notre Théâtre est un des plus grands ressorts de nos préventions contre l'ancien, il a fallu dans un troisième Discours faire voir l'étendûë $\&$ les bornes de la comparaison entre le Théâtre antique \& le moderne, établir des principes, en tirer des conclusions, \& fonder le parallèle sur le caractère des Siècles $\&$ des Génies, des Poëtes $\&$ des Spectateurs ${ }^{18}$."

Viennent ensuite les traductions intégrales de trois tragédies de Sophocle (EdipeRoi, Électre, Philoctète) et deux d'Euripide (Hippolyte, Iphigénie en Aulide). Les traductions sont précédées de l'exposition du sujet et de la liste des personnages, et suivies de " réflexions » et d'extraits de textes anciens ou contemporains qui sont mis en relation avec la tragédie traduite.

Le deuxième volume est composé d'une part de la suite des traductions d'Euripide (Iphigénie en Tauride et Alceste) et d'autre part de l'examen systématique des trois tragiques. Est d'abord examinée l'œuvre d'Eschyle, résumée : aucune de ses tragédies n’est traduite. Viennent ensuite les résumés des tragédies de Sophocle qui n’ont pas été traduites dans la première partie, puis de celles d'Euripide.

L'absence de traductions d'Eschyle n'a pas manqué de surprendre ${ }^{19}$. Brumoy s'en justifie dès son discours préliminaire, par des considérations philologiques - les textes d'Eschyle sont particulièrement corrompus - et plus encore pédagogiques. Eschyle risquerait en effet de " dégoûter " les lecteurs : "Son extrême simplicité \& ses défauts auraient pû d'abord dégoûter les Lecteurs, trop ou trop peu prévenus en sa faveur. » Et pour appuyer son propos, il cite Tanneguy Le Fèvre et sa critique de la « hardiesse des épithètes » chez Eschyle, s’appuyant donc sur les propos d'un érudit peu soupçonnable

17 Discours sur le théâtre des Grecs, p. XVI.

18 Ibid.

19 Claire Lechevalier, qui s'est intéressée aux traductions du Prométhée enchaîné d'Eschyle, s'est plus particulièrement arrêtée sur l'attitude ambiguë de Brumoy à l'égard de ce poète. Voir C. Lechevalier, L'Invention d'une Origine. Traduire Eschyle en France de Lefranc de Pompignan à Mazon : Le Prométhée enchaîné, Paris, Champion, 2007. 
de vouloir desservir les Grecs, selon un procédé récurrent chez Brumoy quand il s'agit de critiquer les Anciens : "La hardiesse de ses épithètes est telle qu’il est impossible de les représenter en notre langue sans lui faire violence ", écrit en effet Tanneguy Le Fèvre dans son Abrégé des Vies des Poëtes. Mais, ajoute Brumoy, " on n’en connaîtra pas moins ses Euvres par la suite de cet Ouvrage » : la méthode de Brumoy, toute de modestie au service des textes, ne prétend ici à rien de moins qu'à mieux servir les textes d'Eschyle qu'Eschyle lui-même!

Aristophane n'est pas mieux traité. Le troisième volume, qui correspond à la troisième partie, lui est consacré. Comme Eschyle, il n'a droit qu'à des résumés, au mieux avec de larges extraits traduits, mais aussi à un vaste appareil textuel ${ }^{20}$. Le volume s'ouvre par un Discours sur la comédie grecque, suivi de Fastes de la guerre du Péloponnèse et d'une carte dépliante de la Grèce. Viennent ensuite les résumés, plus ou moins développés. Les Oiseaux, Ploutos et les Nuées se voient réserver un sort à part, puisque ces comédies bénéficient de longs passages traduits. Brumoy prend ici la suite de ses prédécesseurs, puisque le Ploutos et les Nuées avaient déjà été traduits par Anne Le Fèvre en 1684 et que la traduction des Oiseaux de Jean Boivin le jeune venait juste d'être publiée, en 1729. Brumoy commence par informer le lecteur sur les conditions de la vie théâtrale à l'époque d'Aristophane; puis des introductions précèdent chaque comédie. On y trouve des indications d'ordre chronologique et historique, la datation, les références aux tragédies parodiées. Des notes enfin explicitent les passages obscurs. L'ouvrage offre donc une introduction à Aristophane et vise une lecture éclairée, sans pour autant donner de traduction intégrale. Enfin, le volume se clôt sur un Discours sur le Cyclope d'Euripide et sur le spectacle satyrique, suivi d'une traduction quasi intégrale du Cyclope.

\section{La méthode de Brumoy : "une nouvelle poétique par les faits"}

Dans une époque encore marquée par la Querelle, regrouper l'ensemble des œuvres dramatiques grecques risquait de passer pour une prise de position en faveur des Anciens. Brumoy ne s'interdit d'ailleurs pas de critiquer le théâtre moderne ${ }^{21}$.

20 Édith Flamarion s'est interrogée sur le traitement que Brumoy réserve à Aristophane dans le Théâtre des Grecs. Voir É. Flamarion, "Que faire ? ", 1999.

21 Ainsi à propos de la galanterie : "Supposons aussi qu'Euripide revînt à son tour de l'autre monde, \& qu'il assistât à la représentation d'Iphigénie de M. Racine, sans parler des autres spectacles [...] que dirait-il, je ne dis pas de l'Episode d'Eriphile, espèce de duplicité d'action \& d'intérêt inconnuë aux Grecs, mais de la galanterie Françoise d'Achille, beaucoup plus ignorée d'eux ? [...] que dirait-il des entretiens seuls à seul d'un Prince \& d'une Princesse? ", Discours sur le Théâtre des Grecs, p. XI. 
Pourtant, les intentions de Brumoy sont plus complexes. Il ne s'agit pas d'exalter les Grecs pour dénigrer le théâtre de son temps, à la manière des jansénistes qui prônent une fidélité absolue au texte par refus non seulement du siècle mais du monde. Le jésuite affirme au contraire la nécessité d'un contact des œuvres avec le monde, et donc qu'il faut les soumettre à la critique. Mais pour que cette critique soit juste, il faut au préalable effectuer tout un travail pédagogique visant à les rendre accessibles. Les textes antiques doivent donc être communiqués de la façon la plus large possible, et pour les faire vivre, il faut faciliter leur réception. C'est là que réside toute la singularité de la "méthode Brumoy ".

De cette position découle en effet une méthode originale, qui procède d'un souci de réhabilitation des Tragiques grecs ${ }^{22}$. Partant du constat qu' " on ne connaît presque plus le théâtre des Grecs ", Brumoy s'efforce d'abord de comprendre cette méconnaissance, puis d'y remédier. Ce discrédit dont souffrent les Tragiques grecs - car il est plus question de tragédie que de comédie dans cet ouvrage - Brumoy l'attribue à l'esprit cartésien qui règne sur son temps :

"Le génie philosophique de Descartes... nous a fait croire peu à peu que nous avions chez nous des thrésors asses estimables pour nous passer des richesses étrangères, sur-tout quand il les faut acheter par de pénibles voyages. Cet esprit, ami de l'indépendance, en renversant d'abord la Philosophie ancienne, puis en nous faisant les arbitres suprêmes de tout art et de toute science, sans égard au poids de l'authorité, nous inspire je ne sçai quel dédain pour tout ce qui se refuse à l'examen de nos lumières. Il est plus court \& plus aisé d'estimer peu, ou même de mépriser ce qui coute trop à connoître : \& les débris du Théâtre ancien paroissent trop scabreux pour achetter un simple plaisir de goût par une peine qu'on ne croit pas devoir être asses dédommagée."

Mais il ne s'agit pas pour autant de renoncer à l'esprit cartésien, bien au contraire. Brumoy se propose, loin de tout argument d'autorité, mais aussi de tout préjugé de mépris, de juger les Tragiques grecs " par ses lumières » :

"Mon dessein est de les tirer, du moins en partie, des ténèbres où nous paraissons les avoir condamnés, \& de les citer de nouveau au tribunal, non du petit nombre, mais du Public; non pour arracher l'approbation en leur faveur, ou les livrer à la censure, mais afin qu'ils soient jugés avec quelque connoissance de cause, sans égard aux authorités favorables ou contraires, \& avec l'esprit Cartésien, autant qu'il peut s'appliquer aux choses de pur goût $\mathrm{t}^{23}$."

22 Cette méthode est très bien analysée dans E. FLAMARION, "Que faire ?", 1999, dont nous résumons ici le propos.

23 Discours sur le Théatre des Grecs, p. IV. 
C'est donc « l'esprit cartésien » que Brumoy propose de mettre en œuvre pour juger les Anciens ; mais à la raison il propose d'ajouter la curiosité et, pour ainsi dire, une forme de relativisme. Car pour juger sans préjuger un "ouvrage de goût ", il faut pratiquer le décentrement. Le Théâtre des Grecs devient ainsi une invitation au voyage, temporel et culturel. Pour goûter les Tragiques grecs, il faut en effet que le lecteur "devienne Athénien » et, à propos de l'Alceste d'Euripide, Brumoy écrit :

"Si à mon tour par un effort d'imagination que je lui dois, je me transporte au Thêâtre d'Athènes pour voir agir ses Acteurs, $\&$ me prêter à tous le spectacle, sans faire attention que je lis (car une Tragédie n'est point faite pour être luë, elle est toute action); enfin si Alceste renferme les principales conditions que le bon sens exige dans un Poëme de cette nature, \& si je deviens Athéniens, comme ceux que le Poële a eu en vûe de réjouir, je ne puis m'empêcher, malgré quelques défauts que j’apperçois avec le Parterre, de joindre mes applaudissements aux acclamations de la Grece assemblée, puisqu'étant homme comme les Grecs, je suis nécessairement touché des mêmes vérités $\&$ des mêmes beautés qui ont frappé si vivement leurs esprits ${ }^{24}$.»

C'est à la fois au nom d'une humanité partagée, par-delà la distance temporelle et culturelle, et par un effort de décentrement pour abolir cette distance que le théâtre grec peut encore être apprécié. Cette relativité nécessaire, Brumoy l'explique en transposant la distance temporelle qui le sépare des Grecs au plan géographique, pour opérer un véritable retournement de valeurs :

"En un mot, si semblable à un Chinois qui se trouverait tout à coup présent à une Cérémonie Turque, je trouve tout cela risible, [...], les Spectateurs Grecs n’auraient-ils pas droit de rire eux-mêmes de mon étonnement, \& de dire : Quelle est donc votre idée ? De quel monde venés-vous ? que trouvés-vous en ceci de si étrange, \& que voies-vous sur le Thêâtre, que vous ne retrouviés dans Athènes ? Ils auraient raison sans doute, et peutêtre n'aurais-je pas tort ${ }^{25} \ldots \%$.

Il faut donc, pour "se mettre en situation de les juger avec quelque sorte d'équité ", accepter de se décentrer. Pour faciliter ce décentrement nécessaire, Brumoy se propose d'accompagner son lecteur, tel un guide dans un pays étranger. Refusant toute forme d'érudition, il ambitionne de " faire trouver beau le pais » dont il parle, et donc de faire un récit de voyage aussi séduisant que l'objet de son récit. À cette fin, outre les textes, tout un appareil critique accompagne le lecteur :

« Il a fallu nécessairement des Notes pour l'intelligence du Texte. J'en ay mis quelquesunes ; mais le moins \& les plus courtes qu'il m’a été possible, persuadé qu'une Pièce de 
Théâtre doit être lue de suite $\&$ sans interruption si l'on veut en sentir le Tragique \& en voir l'œconomie ${ }^{26}$."

À nouveau, le souci d'éviter l'érudition guide le jésuite. Plus curieusement, c'est ce même souci de ne pas perdre son lecteur qui l'amène à ne pas traduire toutes les pièces intégralement :

"Je n'ai pas crû qu'il fût possible de traduire tout au long la plupart des Tragédies Grecques ; \& je doute qu'en ceci Mr \& Madame Dacier eussent tenu la parole qu'ils semblaient avoir donnée au public. Ils auraient été rebutés non seulement par le préjugé invincible contre quelques fictions \& certaines coutumes anciennes trop choquantes pour nous, mais encore par un très grand nombre de morceaux dont toute la beauté consiste précisément dans l'expression originale : tels sont la plûpart des chœurs. L'urbanité françoise ne peut rendre leur atticisme. C'est comme si l'on voulait tourner nos chansonnettes en Grec. Un tour en toute langue vaut souvent une pensée, \& en est véritablement une. Mais c'est une manne qui fond, un fantôme qui s'évanouit, ou du moins une fleur qui se fanne dans une langue étrangère ${ }^{27}$. "

Deux obstacles majeurs s'opposent donc à une traduction complète : certaines coutumes des Anciens, considérées comme trop choquantes, et les chœurs. On peut néanmoins s'interroger sur la bonne foi de ces allégations : Brumoy traduit Edipe-roi de Sophocle, qui n'est pas la moins choquante des tragédies. Quant aux chœurs, il traduit bien ceux des sept pièces qu'il donne intégralement... Paresse de traducteur ? Ou souci d'expliquer plutôt que d'exposer, au risque de lasser ? Il fait le choix étrange de substituer aux pièces non pas tant des résumés que ce qu'il appelle des " analyses raisonnées" :

"J’y ai supplée en prenant une route peu différente, \& peut-être plus agréable, \& non moins instructives ; je veux dire par des analyses raisonnées, où presque tout est traduction, où nul trait considérable n'est omis, où enfin le Poête se fait autant connoître que dans une traduction suivie... une analyse qui est faite avec soin et qui nourrie du suc du poète présente les principaux endroits du poème avec tout son plan coûte souvent plus que la traduction même, \& peut faire autant d'impression que la pièce dont on veut donner l'idée... le dirai-je ? quelquefois elle ennuie moins ; et pour le dire encore, il est bien des lecteurs que certains pièces de l'Antiquité théâtrale, exposées trop, auraient ennuies après avoir diverti Athènes ${ }^{28}$."

Il s'agit donc d' " extraire le suc " des poètes pour mieux les servir : donner le plan des pièces, traduire ce qui peut l'être et éviter à tout prix l'ennui des lecteurs. Pour la comédie, il va jusqu'à faire un véritable cours d'histoire :

26 Ibid., p. XX.

27 Ibid., p. XXI.

28 Ibid., p. XXI. 
"On prépare ensuite le Lecteur à ce qu'on peut lire d'Aristophane, par des Observations nécessaires, \& par les Fastes de la guerre du Péloponnese [...] Dans les détails des Pièces on explique tous les évenements historiques [...] et l'on traduit tout ce qui peut être traduit, en se proposant quatre principaux objets qu'on remet devant les yeux : particulièrement le Gouvernement d'Athènes dévoilé dans les allégories du poète $\&$ le génie de la comédie antique. [...] En un mot on a tâché de ne rien omettre pour faire connaître à fond Aristophane ${ }^{29}$."

\section{Le comparatisme de Brumoy: le souci de la mise en relation des pièces}

C'est dans ce même souci pédagogique que s’inscrit le comparatisme de Brumoy, qui rassemble en réalité dans son ouvrage beaucoup plus que le théâtre grec :

"La nature des ces Analyses \& le défit de faire connoître à fond le Théâtre Grec m’ont porté à recueillir en chemin et à enchâsser en passant tout ce que j'ay trouvé y avoir quelque conformité, comme des traits d'histoire, des pensées de divers poètes, des caractères $\&$ des tours imités exprès ou par hasard ${ }^{30}$."

Brumoy replace ainsi le théâtre grec dans une histoire continue ; il s'efforce de combler les zones d'ombres et d'atténuer la rupture apparente entre ce théâtre et le théâtre moderne, ce qui l'amène à excéder les bornes du théâtre grec pour finalement envisager l'histoire du théâtre dans son entier, en ayant à cœur de mettre en lumière " cet enchaînement suivi, de traductions, de critiques, de raisonnements, \& de comparaisons de goût, qui compose une sorte d'histoire du Génie Théâtral, \& une nouvelle espèce de Poétique par les faits ${ }^{31}$ ».

Et tout comme il tente de rétablir les liens entre théâtre antique et théâtre moderne, il s'efforce d'envisager le théâtre grec comme un tout organique, certes mal en point mais qu'il convient de reconstituer pour le faire apprécier :

"Pour former une idée précise et complète du théâtre ancien, il fallait en recueillir tous les restes ; faire un assemblage suivi, comparer les œuvres de chaque poète entre elles, chacun d'eux avec les rivaux ; saisir par cette comparaison leur caractère et leur génie ; en marquer avec justesse les traits généraux et particuliers, même les plus délicats ; réunir, confronter, assortir, lier_les parties, en composer un tout ; débrouiller le chaos pour en

29 Ibid., p. XXV.

30 Ibid., p. XXII.

31 Ibid., p. XXV. 
tirer un corps vivant et animé avec ses justes proportions ; en un mot, rebâtir le théâtre ancien de ses propres débris ${ }^{32}$."

Le théâtre grec est ainsi comparé à un corps, dont les œuvres constituent les différents membres. Il s'agit donc certes de traduire les œuvres - même si l'on a vu que ça n'était finalement pas l'essentiel - mais aussi et surtout, pour leur redonner vie, de restituer leur contexte et mettre en lumière leurs liens - d'où les discours préliminaires et les notes abondantes accompagnant ces textes. Et comme ces œuvres forment un tout, il convient de ne faire aucun choix, et de comparer les poètes entre eux pour les "faire connaître les uns par les autres".

\section{Le goût de l'expérience et de l'observation}

Observer, exposer, expliquer, comparer puis, seulement, juger : Brumoy semble s'inspirer du goût de son siècle pour l'expérience, y compris en matière de goût. L'observation doit précéder la théorie, et donc " il faut montrer les Anciens tels qu'ils sont ${ }^{33}$ ". Charalampos Orfanos montre dans les pages qui suivent l'influence de ce souci de l'observation dans la manière dont Brumoy appréhende la Grèce. Laisser la parole aux Anciens, tel est le mot d'ordre :

" Je ne dirai rien par moi-même. Les Poëtes parleront pour eux. On a tant écrit sur le Théâtre, qu'il semble difficile de rien dire de nouveau. Mais on ne l'a point encore fait, que je sache, de la manière dont j'entreprends de le faire aujourd'hui. On a donné beaucoup à la théorie sur les traces d'Aristote, \& même à la Pratique, comme M. L'Abbé d'Aubignac. Il y manquait d'exposer le Théâtre ancien dans le point où il faut l'envisager, pour le bien connoître, c'est-à-dire en lui-même, par l'exposition des œuvres Tragiques \& Comiques, jointe à la manière dont elles ont été composées, \& aux conjonctures des lieus et des temps qui en sont inséparables ${ }^{34}$."

Mais si l'on se place du point de vue de la réception du théâtre grec, force est de constater qu'en faisant du théâtre grec un tout imaginaire, alors même qu'il ne dispose que de débris, il en donne une lecture téléologique et la diffuse durablement. Et le projet de " montrer les Anciens tels qu'ils sont " est bien entamé par la pratique abondante des analyses raisonnées.

32 Ibid., p. XIV.

33 Ibid., p. XXVII.

34 Ibid., p. XXVII. 


\section{Brumoy traducteur}

Cependant, s'il ne traduit pas tout, Brumoy traduit. Mieux, il réfléchit à sa pratique de la traduction, qu'il expose également dans son discours préliminaire. Il s'agit pour lui d'emprunter une voie moyenne, d'adopter une esthétique du juste milieu " entre l'exactitude trop scrupuleuse " et " la licence qui [les] altère 35 ". Ce sont tout d'abord les partisans des Anciens qui sont visés, et plus précisément les auteurs de traductions ad verbum, car " toute langue a ses arrangements d'idées, ses tours \& ses mots, nobles ou bas, énergiques ou faibles, vifs ou languissants. C'est un principe qu'on ne saurait nier. Qui voudrait traduire les anciens mot pour mot en François, \& suivant le tour Grec, les travestirait sans doute, \& les rendrait ridicules à peu de frais. Voilà le premier degré de cette fausse fidélité dont je parle ${ }^{36}$ ".

Mais les Modernes ne sont pas épargnés, et Brumoy semble profiter ici du sujet pour s'en prendre à Perrault et à ses parodies qui consistent à " changer les expressions reçues dans le bel usage de l'antiquité, en termes bas $\&$ populaires, comme le faisait Mr. Perrault ${ }^{37}$ \%.

Et alors qu'il annonçait suivre la voie du juste milieu entre deux extrêmes, il rejette une troisième voie, qui n'est autre que celle suivie par ses récents prédécesseurs, et notamment Perrot d'Ablancourt pour Homère :

«Le troisième degré, c'est de s'asservir scrupuleusement à exprimer toutes les Epithetes, $\&$ à faire d'un beau mot Grec une méchante phrase françoise, ou un allongement vicieux qui amortit le feu des Poëtes... On doit à l'équité de leur faire parler François (autant qu'on le peut) comme ils parleraient eux-mêmes, s'ils faisaient passer leurs pensées en notre langue. Pourquoi changer en monnoie de cuivre un dépôt que l'on peut conserver en or ? La versification ancienne se rend heureusement par une prose poétique, qui joint ses grâces à celles des vers anciens ${ }^{38}$. "

Brumoy critique donc ici les traductions en vers au profit de la "prose poétique ». Mais sa construction à trois termes est bancale : les parodies de Perrault ne sont pas des traductions, et la traduction ad verbum, très en vogue en latin au XVI ${ }^{\mathrm{e}}$ siècle, ne se pratique plus. Brumoy semble s'inventer des repoussoirs pour justifier son projet, qui ne fait peut-être pas beaucoup plus que prolonger les travaux de ses récents prédécesseurs, dont il cherche néanmoins à se distinguer. Perrot d'Ablancourt et surtout André Dacier sont ainsi critiqués, avec l'idée que les traductions des poètes requièrent des qualités spécifiques et que la seule érudition ne suffit pas :

35 Ibid., p. XVI.

36 Ibid., p. XVII.

37 Ibid., p. XVII.

38 Ibid., p. XVII. 
" Je rends justice à l'érudition de leurs Traducteurs. Mais je crois aussi devoir quelque chose à la vérité. Il faut plus d'âme $\&$ de génie pour tourner ces sortes d'ouvrages, que pour manier des Euvres Philosophiques. Le feu soutient jusqu'aux défauts, \& la langueur fait expirer les Graces mêmes. J'aimerois mieux faire passer dans le style, fût-il négligé, tout l'enthousiasme des Poêtes Grecs, que de leur donner un air froid à force d'être concerté. Une traduction froide est un visage de cire ${ }^{39}$. "

Il recourt alors à l'image du portrait : la traduction des poètes est au texte original ce qu'est le portrait au modèle, et le traducteur est donc, implicitement, comparé à un peintre. La traduction poétique est alors considérée comme un art, et le peintre se doit d'avoir du talent pour traduire la vie qui habite son modèle ${ }^{40}$.

C'est donc une véritable conception de la traduction qui est ici exposée, que Brumoy distingue de la pratique de ses prédécesseurs, avec une relative mauvaise foi : on trouve des arguments assez proches dans les propos des Dacier, qui eux aussi se targuaient de ne pas faire du " mot à mot " à la manière des traducteurs du $\mathrm{XVI}^{\mathrm{e}}$ siècle.

\section{L'horizon de la scène}

Si elles ne sont pas traduites pour la scène, les tragédies que donne Brumoy ont pourtant la scène comme horizon. L'intérêt de Brumoy pour le théâtre des Grecs est en effet lié à la pédagogie des Jésuites pour qui la tragédie peut servir à former les mœurs. Ils different en cela de leurs contemporains qui condamnent largement les plaisirs du théâtre, soupçonné de susciter des passions incontrôlables. À l'inverse Brumoy, tout comme Porée dans le De Theatro que Brumoy traduit en 1733, assigne à la tragédie grecque une dimension morale : elle permettrait de "s'apprivoiser avec l'idée des maux et de se fortifier contre eux ".

Dans cette perspective, la scène joue un rôle prépondérant, et elle n'est donc pas absente des préoccupations de Brumoy, comme le montrent les contributions de Claire Lechevalier et Jean-Philippe Grosperrin. Les traductions sont faites en fonction de leur possible mise en scène, les didascalies sont abondantes - d'où également un style rapide et une tendance à l'omission, par souci de l'esprit du texte plus que de sa lettre.

\section{Brumoy, suite et fin ?}

Le Théâtre des Grecs connaît immédiatement un grand succès et il est aussitôt réimprimé : en 1732 à Amsterdam, cette fois en petit format (six volumes in-12) puis

39 Ibid., p. XIX.

40 Ibid., p. XIX. 
en 1749 à Paris, toujours en in-12. En 1763 paraît une version " revue, corrigée et augmentée ", dont les ajouts concernent surtout les notes. Tel n'est pas le cas pour l'édition de 1785-1789, qui se présente comme la première édition " complète ", que l'on doit à Rochefort et du Theil. En treize tomes in-8, elle offre en effet la traduction de toutes les tragédies grecques et d'Aristophane. Y figurent également un quatrième discours, sur la tragédie grecque, et une Vie d'Euripide. Enfin à partir de 1820 paraît une " seconde édition complète » due cette fois à Désiré-Raoul Rochette, qui augmente la somme d'un cinquième discours sur l'origine de la tragédie et de la comédie grecques et surtout des fragments de Ménandre et Philémon.

Ce succès excède les frontières de la France : le Théâtre des Grecs est traduit en anglais, lu en Allemagne et en Italie. En France, Rollin fait un grand éloge du P. Brumoy, qui l'a "bien aidé ", dans le tome $\mathrm{V}$ de son Histoire ancienne où " il parle des jeux, de la Grèce, des spectacles, du poème dramatique, de la tragédie, de la comédie, des poètes ». Jean-Baptiste Rousseau (1670-1741) lui rend hommage dans son Épitre au P. Brumoy ainsi que Goujet dans sa Bibliothèque françoise. Seul Voltaire formule des réserves et se montre sévère avec les traductions de Brumoy, auxquelles il attribue des erreurs. L'ouvrage n'en connaît pas moins, de réimpressions en rééditions, une postérité impressionnante, comme le montre la contribution de Jean-Noël Pascal.

Nous proposons ici un parcours qui mènera le lecteur de considérations portant sur la méthode de Brumoy (Charalampos Orfanos), à des réflexions sur un aspect plus particulier de son approche du théâtre grec (la question de la "cérémonie " avec JeanPhilippe Grosperrin, puis celle de la représentation avec Claire Lechevalier). Enfin sera abordée la question de la réception, que l'on sait considérable, de Brumoy, avec notamment son rôle dans l'interprétation satirique d'Aristophane qui prévaudra après lui (Romain Piana) et une mise en regard de ses analyses sur la tragédie avec celles de son contemporain Luigi Riccoboni (Filippo Fassina). Nous finirons alors, avec JeanNoël Pascal, sur une présentation analytique des rééditions du Théâtre des Grecs.

Malika Bastin-HaMmOU

Université Stendhal-Grenoble III

Malika.Bastin@u-grenoble3.fr

2 rue Auguste Gaché

38000 Grenoble 